\section{THIS}

EDITORIALS
DATABASES Clinical-trial efforts should enforce registration rules $\mathbf{p . 2 9 2}$

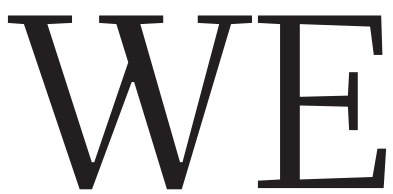

WORLD VIEW Why we pushed Mendel off the curriculum $\mathbf{p . 2 9 3}$
GENETICS Shifting sands maintain ancient camel genome $\mathbf{p . 2 9 5}$

\title{
Zika must remain a high priority
}

\author{
Although the evidence suggests that the Olympic Games are safe to proceed, the global health \\ community must not let the Zika virus fade from the research agenda until the threat is wiped out.
}

A re this summer's Olympic Games under threat from the Zika virus? Or, more importantly perhaps, are the competitors and spectators? Athletes such as US women's soccer goalkeeper Hope Solo have said they will go "begrudgingly", and will barely leave their hotel rooms. Some have called for the event to be cancelled.

This overestimates the risk of Zika to visitors. Although publichealth agencies have advised pregnant women to avoid countries with active Zika transmission owing to the threat of birth defects, there are much more pertinent threats to the average visitor to Brazil, including dengue virus and random street violence. Much remains unknown about Zika, but a great deal is known, too, and it suggests that there is no reason to cancel the event.

That we know so much so soon is a victory. When the US Centers for Disease Control and Prevention (CDC) concluded last month that the Zika virus causes birth defects, that marked the conclusion of one of the quickest-ever basic-science investigations into a crucial publichealth issue. Some researchers expected it to take years. In the end, it took just six months.

The CDC cited a growing number of studies using a range of approaches, including epidemiological, molecular and pathological, that showed a link between Zika and birth defects. The wisdom of making such a declaration so quickly remains a matter of some debate, but it is noteworthy that the global scientific community was able to organize itself in a relatively rapid fashion. There are three main reasons why this happened, and they offer lessons for research in future outbreaks.

First, Brazil has a substantial amount of research infrastructure. Unlike some regions where outbreaks occur, such as nations in Africa, Brazil has made sizeable investment in research and public health. It is the only country in Latin America that invests more than $1 \%$ of its gross domestic product into research, and the country is well supported by foreign research institutes, such as the US National Institutes of Health (NIH). The country spent US $\$ 31.9$ billion on research in 2013, for instance.

This meant that when the Zika outbreak arose, there was a highly trained group of researchers ready to investigate the issue. The Oswaldo Cruz Foundation, a federally funded network of research stations across Brazil, has led investigations of the epidemiology and molecular aspects of Zika. And a long-running NIH-funded project on urban slum health in northeastern Brazil quickly adapted to set up large cohort studies to look at the possible link between Zika and birth defects.

Second, there have been significant advances in neuroscience. Some of the most telling evidence for Zika's effects on human brain cells has come from studies of human neural stem cells and organoids - brain-like structures that can be grown in cell culture in the lab. Brain organoids were developed only a few years ago. By using organoids, human stem cells and human fetal tissue, researchers were

quickly able to show that Zika preferentially targets and kills human neural precursors in the brain, offering a plausible explanation for a mechanism through which the virus causes birth defects.

Third, the global health community has acknowledged that its dismally slow response to the Ebola epidemic let it grow out of control. Health officials were therefore determined to move more quickly in response to Zika. The World Health Organization declared the out-

"There

should be no complacency."

approve that spending, the CDC said on
13 May that it had found $\$ 85$ million to distribute to states to help them prepare for the virus.

There should be no complacency. The quick connection of dots between Zika and birth defects is only the beginning of the necessary mobilization. And we still don't know why only some babies succumb to the devastating effects of Zika, or the most effective way to care for them. The questions will keep coming, and the research community and funders must continue to pursue them - with speed, strength and the highest urgency, as the organizers of the Olympics might say.

\section{Second thoughts}

Revisiting the past can help to inform ideas of the present.

1 The thought experiment has a noble place in research, but some thoughts are deemed more noble than others. Darwin and Einstein could let their minds wander and imagine the consequences of certain actions or natural laws. But scientists and historians who try to estimate what might have happened if, say, Darwin had fallen off the Beagle and drowned, are often accused of playing parlour games.

Most of these counterfactual thought experiments tend to focus on changes to the lives of historical figures - what would have happened had Hitler never been born, for instance. Dismissed as silly and speculative, such exercises are considered of little academic value, because the results of the experiment tend to align with what the experimenter would have wanted to happen. (One of first such published accounts seems to prove the point: Napoléon Apocryphe, published by a supporter in 1841, starts with the emperor surviving the 1812 Russian winter in an unburnt Moscow, conquering Europe, then Asia, Africa and the Americas, while discovering a new planet and inventing a flying car on the way.) 
The course of history is surely contingent on the roles of influential individuals, which is why counterfactual tales of thwarted plots succeeding and dictators killed as infants seem so poorly anchored to reality. An individual really can steer events, so a world without that individual is unknowable. But what about the course of science, and the ideas that push it along? Is scientific and technical progress equally contingent on circumstance and personality? Or are discoveries inevitable, and independent of the people who happen to be around to make them?

What if Darwin had toppled overboard before he joined the evolutionary dots? That discussion seems useful, because it raises interesting questions about the state of knowledge, then and now, and how it is communicated and portrayed. In his 2013 book Darwin Deleted — in which the young Charles is, indeed, lost in a storm - the historian Peter Bowler argued that the theory of evolution would have emerged just so, but with the pieces perhaps placed in a different order, and therefore less antagonistic to religious society.

On page 293, another historian offers an alternative pathway for science: what if the ideas of Gregor Mendel on the inheritance of traits had been challenged more robustly and more successfully by a rival interpretation by the scientist W. F. R. Weldon? Gregory Radick argues that a twentieth-century genetics driven more by Weldon's emphasis on environmental context would have weakened the dominance of the current misleading impression that nature always trumps nurture.

Unusually for a historian, Radick has some experimental data to

draw from. Over a term, students at the University of Leeds, UK, where Radick teaches, were presented with a curriculum that relegated Mendel to the margins and promoted Weldon in his place. The result, perhaps not surprisingly, was a class of undergraduates who were less

\section{"Science without} consensus would be chaos."

formed at all. If the past is a foreign country,
then it is also supposed to be one that cannot be revisited. With a little imagination, what other thought experiments could be tested in this way? The history of science is, after all, littered with major theories that became scientific orthodoxy but initially attracted a great deal of toughminded criticism, from the Newtonian theory of universal gravitation to the Copenhagen interpretation of quantum mechanics.

These 'winners' became dominant before all the criticisms against them were fully answered, which raises questions about why the debates went the way they did, and whether they could have gone otherwise - and if so, with what repercussions.

A well-informed interest in alternative scientific pasts can help us to take the actual past more seriously as a source of present-day insight. It can also help us to stay self-critical as we make choices in the present. Science without consensus would be chaos. But the price of consensus is eternal vigilance against complacency, and a willingness to contemplate the road otherwise not travelled.

\section{Open medicine}

\section{Governments need to tighten regulation if the sharing of clinical-trial data is to succeed.}

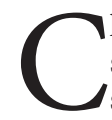

linical science has a compatibility problem. Although there are set protocols to test medicines and to treat patients, no such standards exist to compare clinical-trial data.

The problem arises because each research group has a preferred method of collecting and categorizing results. Differences can be as great as omitting or including the gender and ethnicity of patients enrolled, or as mundane as the vocabulary used in medical records. For example, a study published in The BMJ this week challenged the 'weekend effect' the idea that people in the United Kingdom admitted to National Health Service hospitals at weekends are more likely to die compared with those admitted on weekdays - by saying that stroke patients' conditions are frequently miscoded by UK hospital staff (L. Li et al. Br. Med. J. 353, i2648; 2016). If proved, such a finding could cast doubt on research studies that use medical records as a data source.

The lack of a single place to report descriptive metadata compounds the problem. Data sharing is most challenging for developing countries, which often lack the resources for large-scale statistical analysis (L. Merson et al. N. Engl. J. Med. http://doi.org/bhmb; 2016).

The latest attempt to address this problem is Vivli, described last week in The New England Journal of Medicine as a universal platform to "link existing data-sharing platforms and communities, while hosting data from investigators who aspire to share data but lack the resources to do so" (B. E. Bierer et al. N. Engl. J. Med. http://doi.org/ bhmc; 2016).

The platform, to be run by the Multi-Regional Clinical Trials Center of Brigham and Women's Hospital and Harvard in Cambridge, Massachusetts, will initially employ curators to convert the clinical-trial data they receive from researchers into a standard, anonymized format and post it on the platform for other researchers to access on request. Eventually, the system's creators hope to switch to automated curation.

Vivli's creators acknowledge its limitations. It will no doubt be

useful for researchers to verify findings, avoid duplicating trials and prioritize next steps. Similar efforts by scientific societies and researchers on specific diseases have made progress in these directions. But data anonymization and standardization is time-consuming, and so only limited amounts of medical data will at first be available.

Yet the best database in the world cannot address the underlying and pernicious deficiency in clinical-trial data sharing. Unless government regulation is rapidly and substantially changed, Vivli will lack data from the majority of clinical trials - leaving it far from the comprehensive database that its creators envisaged. Despite US laws requiring trial data to be publicly disclosed in the ClinicalTrials.gov database, lax enforcement, loopholes and ambiguous interpretation have allowed pharmaceutical companies to selectively report positive results, alter endpoints and avoid publishing data on time.

Most trials disappear altogether. Some studies estimate that only one-third of trials for drugs approved by the US Food and Drug Administration (FDA) are ever published (J. S. Ross et al. PLoS Med. 6, e1000144; 2009). One assessment found that only two of the ten large pharmaceutical companies complied with FDA regulations on data reporting for drugs approved in 2012 (J. E. Miller et al. BMJ Open 5, e009758; 2015). Gilead and Sanofi Pharmaceuticals were the worst offenders according to author Jennifer Miller of Bioethics International. And private industry is not the only offender about one-third of federally funded trials remain unpublished four years after completion (J. S. Ross et al. Br. Med. J. 344, d7292; 2012).

Hidden clinical-trial data have long been an open secret in the field, but researchers are now starting to assess it more quantitatively. On 17 May, the Laura and John Arnold Foundation awarded Bioethics International a US\$3.6-million grant, which will allow the organization to assess trial-data publication, or lack of it, for all new FDA-approved drugs and biologics.

Ideally, knowledge of such poor compliance will shock the public, pushing the FDA and other regulators into creating stronger incentives for companies to publish complete data, such as serious

$\rightarrow$ NATURE.COM To comment online, click on Editorials at: go.nature.com/xhunqv fines and penalties. Without such intervention, well-intentioned efforts such as Vivli are doomed to remain tragically incomplete, robbing researchers of the opportunity to truly link their work. 